\title{
Germanica
}

\section{Ernst Barlach: Die Sündflut (1924) Essai d'approche théologique}

Ernst Barlach: Die Sündflut (1924)

Bernard Bach

\section{OpenEdition}

\section{Journals}

Édition électronique

URL : http://journals.openedition.org/germanica/2248

DOI : $10.4000 /$ germanica. 2248

ISSN : 2107-0784

Éditeur

Université de Lille

\section{Édition imprimée}

Date de publication : 1 janvier 1999

Pagination : 29-53

ISBN : 2-913857-00-0

ISSN : 0984-2632

Référence électronique

Bernard Bach, « Ernst Barlach: Die Sündflut (1924)

Essai d'approche théologique », Germanica [En ligne], 24 | 1999, mis en ligne le 01 janvier 1999 , consulté le 06 octobre 2020. URL : http://journals.openedition.org/germanica/2248 ; DOI : https:// doi.org/10.4000/germanica.2248

Ce document a été généré automatiquement le 6 octobre 2020.

(c) Tous droits réservés 


\title{
Ernst Barlach: Die Sündflut (1924) Essai d'approche théologique
}

\author{
Ernst Barlach: Die Sündflut (1924)
}

\section{Bernard Bach}

1 Selon Tillich, la " préoccupation ultime se manifeste dans la fonction esthétique de l'esprit humain sous la forme du désir illimité d'exprimer le sens ultime des choses $»^{1}$. Or le fait d'être saisi par une préoccupation ultime, c'est justement la religion, comprise en un sens large comme "expérience de l'absolu» et dépassant par conséquent le cadre des systèmes constitués. Il y a donc entre religion et culture un lien très étroit. Tillich définit les rapports entre religion et culture comme des rapports de contenu à forme : « La religion est la substance de la culture et la culture est la forme de la religion $»^{2}$. Ainsi la religion en tant que préoccupation du sens ultime «se manifeste dans toutes les fonctions créatrices de l'esprit humain $»^{3}$. Dans cette optique, les créations littéraires, le langage poétique, ont une fonction symbolique, renvoyant audelà d'eux-mêmes à l'Inconditionné, à l'Absolu. Dans la mesure où toute création culturelle recèle cette dimension de sens inépuisable, elle peut faire l'objet d'une approche théologique comprise comme interprétation de la préoccupation ultime dans une œuvre.

2 Die Sündflut de Barlach est une réécriture de l'histoire bien connue de Noé (Gn 6-9). L'interrogation sur ce symbole religieux central de la Bible qu'est Dieu et sur le rapport de l'homme à ce Dieu renvoie à la question du sens même de l'existence humaine et du monde. Derrière cette préoccupation ultime se profile aussi la question de l'espérance ou de la désespérance de l'homme face à son destin. Noé est le héros du déluge et de la première alliance de Dieu avec les hommes. Dans la tradition biblique, le « consolateur " 4 , apparaît de manière continue comme le modèle du juste par la foi ( $\mathrm{Si}$ 44,17-18; Hé 11, 7) sauvé du cataclysme par sa confiance en la parole divine. Dans le discours allégorique de Philon, il représente le juste et la vertu de justice ${ }^{5}$. Dans la tradition des pères de l'Église, l'arche apparaît comme le symbole de l'Église et Noé comme la préfiguration de Christ ${ }^{6}$. Si la conception traditionnelle de Noé est encore reprise au XVII et XVIII ${ }^{e}$ siècle par J. van den Vondel (Noah, Drama, 1667) et JJ. Bodmer 
(Noah, Heldengedicht, 1750-52), Byron, au XIX ${ }^{e}$ siècle, remet en question la soumission de Noé à la volonté divine dans son mystère inachevé Heaven and Earth. A Mystery (1823). Des auteurs comme Alfred de Vigny (Le déluge, 1823), Victor Hugo (Les sept cordes dans Toute la Lyre, 1856), Leconte de Lisle (Quaïn, 1869) refusent l'interprétation orthodoxe, conforme à celle de la Bible, et soulignent notamment le scandale d'un Créateur qui détruit sa propre création. C'est le signe d'un changement d'état d'esprit qui doit être mis en relation avec le mouvement de sécularisation, qui accompagne l'émergence et l'affirmation de l'esprit moderne, et avec l'un des principes fondamentaux du siècle des Lumières, celui de l'autonomie de l'homme. Le Noé de Barlach participe de l'esprit des temps modernes. L'auteur lui-même a confié un jour qu'il avait voulu démontrer l'absurdité du récit biblique ${ }^{7}$. Sans doute serait-il plus juste de dire que Barlach démontre l'absurdité d'une certaine compréhension du récit biblique.

La comparaison du texte biblique et de la pièce de Barlach révèle un déplacement de perspective. L'attention de Barlach se concentre sur le rapport de l'homme à Dieu et sur le Dieu justicier lui-même, il montre que l'un et l'autre sont devenus problématiques au regard des forces d'auto-destruction qui s'emparent de l'homme. C'est en fait toute la compréhension de l'existence humaine face au Dieu courroucé de la Bible qui est remise en question et par conséquent aussi la possibilité d'un espoir de salut pour l'homme face aux forces auto-destructrices qui le menacent. Sans doute le cataclysme de la Première Guerre mondiale n'est-il pas étranger à la manière dont Barlach réécrit l'histoire du déluge. Le changement de perspective entraîne une modification de la structure même de la pièce par rapport au récit biblique. Robert Couffignal $^{8}$ a montré que le récit du déluge avait la forme littéraire d'un conte. Chez Barlach, la structure du conte disparaît, il fait du célèbre épisode un drame en cinq parties qui se termine par l'entrée de Noah dans l'arche et l'arrivée du déluge. L'absence de la fin optimiste, propre au conte, confère au drame de Barlach une tonalité grave, la pièce est dominée par les ténèbres et le désespoir, et s'achève par la destruction de l'humanité, d'une certaine manière par la victoire du non-être. L'absence chez Barlach de la perspective de renaissance de l'humanité et la mise en question de l'attitude de piété filiale de Noé confèrent à la pièce une note de désespérance et de pessimisme qui est absente dans le récit biblique.

\section{L'ambiguiité fondamentale de l'être}

4 Barlach reprend les éléments essentiels du récit biblique avant l'entrée dans l'arche, mais il introduit également des personnages supplémentaires et accentue l'opposition entre les différents groupes. On notera en particulier l'introduction de Calan, riche propriétaire et farouche adversaire de Noah et de son Dieu. A première vue, la pièce semble construite sur l'antagonisme entre Calan, le blasphémateur, et Noah, l'homme pieux : deux façons opposées de concevoir le visage de Dieu et le rapport avec lui. En réalité, la structure de la pièce s'ordonne autour d'une multitude d'oppositions qui placent Dieu, l'homme et le monde sous le signe de l'ambiguïté. Les quatre premières parties (Teile) du drame sont subdivisées respectivement en deux tableaux, la dernière partie comporte cinq tableaux. Les parties I et II sont construites sur la même opposition entre le désert (Wüste) et le monde de Noah (Raum zwischen Noahs Zelten), les parties III et IV sur l'opposition entre le désert et la forêt de montagne (Bergwald) où se construit l'arche. Dans la partie V, le désert disparait au profit d'un lieu unique, la forêt 
de montagne, plongée dans la tempête et l'obscurité. Tout au long de la pièce se dessinent des oppositions fortes à de multiples niveaux : opposition entre le lieu de l'horizontalité (Wüste) et le lieu de la verticalité (Bergwald), entre le lieu inhospitalier, torride, aride et le lieu hospitalier, frais et humide, opposition entre les riches et les pauvres, les malades et les bien portants, ceux qui ont une identité et ceux qui n'en ont pas, ceux qui font la volonté de Dieu et ceux qui agissent contre la volonté de Dieu, ceux qui acceptent leur statut de créature et ceux qui le refusent, ceux qui sont révoltés et ceux qui sont résignés, entre ceux qui dominent et ceux qui sont soumis, entre les bourreaux et les victimes, entre les hommes et les femmes... Les deux protagonistes eux-mêmes sont traversés par des contradictions.

Le monde et les hommes apparaissent sous le signe de l'ambiguïté. Le lieu même du désert, qui est à la fois le lieu de Calan et de Noah, est significatif de cette ambiguïté : dans la Bible, le désert est le lieu de la mise à l'épreuve de l'homme, le lieu des tentations (cf. Le, 4, 1-13; tentation de Noah par Calan I, 2) et le domaine des démons (Mt 4,18$)$ en même temps que le lieu de la rencontre avec Dieu depuis la révélation à Moïse sur le Sinaï (Ex 3). Pour le prophète Osée (Os 2, 16-17), le désert représente l'idéal de pureté et de fidélité originelles. Le choix fait par Barlach de ce lieu hautement symbolique n'est-il pas tout à fait approprié pour signifier l'ambiguïté de l'existence elle-même ? Il en va de même de l'autre lieu dominant de la pièce, la montagne, même si dans la Bible ce lieu n'offre pas la même ambiguïté que le désert. Dans la Bible, la montagne est le lieu de la rencontre avec Dieu (Ex 3,1) et le lieu du culte (Dt 12, 2; 1R $18,19)$. La disparition du désert au profit de la montagne dans la partie $V$ de la pièce est l'expression symbolique d'une décision, celle de Noah, et d'une évolution intérieure, celle de Calan, mais en même temps la montagne reste le heu de la révolte contre Dieu exprimée par le lépreux ainsi que celui de l'hubris manifestée jusqu'au bout par Calan. Significativement, le dernier tableau se place sous le signe du clair-obscur (trübe Helle SÜ 382), manière symbolique de souligner l'ambiguïté même du déluge. Chez Barlach, le désert comme la montagne, les personnages comme les événements sont marqués par l'ambiguïté, celle-ci apparait comme la caractéristique fondamentale de l'existence humaine. Catherine Mazellier-Grünbeck note que «la vérité du personnage de théâtre n'est pas dogmatique, mais artistique $»^{9}$. Du point de vue théologique, la vérité « artistique » est la vérité de l'être même de l'homme sous les conditions de l'existence. L'ambiguïté, c'est l'état d'aliénation qui désigne le fait que l'homme tel qu'il existe n'est pas ce qu'il est essentiellement et devrait être, le fait qu'en lui s'entremêlent de façon ambiguë des puissances d'être constructrices et destructrices. Paul Tillich note que "l'homme est aliéné d'avec le fondement de son être, d'avec les autres êtres et d'avec lui-même » et que «le passage de l'essence à l'existence aboutit à la culpabilité personnelle et à la tragédie universelle $»^{10}$. Pour exprimer ce passage de l'essence à l'existence, la Bible recourt au symbole universel de la chute (Gn 3). La théologie emploie le concept de péché qui souligne davantage «l'élément de responsabilité personnelle » dans l'aliénation ${ }^{11}$. Au fondement de l'aliénation, il y a l'angoisse ${ }^{12}$ qui pousse l'homme à se cramponner désespérément soit au fini, soit à l'infini pour se sécuriser. Enfermé dans le "cercle de l'ipséité » (Sartre), l'homme est incapable de réunifier les deux pôles et de surmonter l'angoisse. Cette ambiguïté fondamentale chargée d'angoisse a une double conséquence qui s'exerce sur la perception de Dieu et sur l'attitude de l'homme: Dieu offre le double visage de Justicier et de Sauveur, toutpuissant et infiniment faible, courroucé et miséricordieux, l'homme rejette et cherche en même temps ce Dieu mystérieux, fondement de l'être. Die Sündflut rend compte de la 
préoccupation ultime qui habite l'homme et de sa manifestation dans les conditions de l'existence. Les visages contradictoires de $\operatorname{Dieu}^{13}$ et les attitudes contradictoires des personnages dans leur rapport à Dieu apparaissent comme la conséquence de l'ambiguïté fondamentale de l'existence.

6 Une lecture théologique de la pièce s'attachera à mettre en évidence les formes que prend ce qu'on pourrait nommer la quête de Dieu, le désir de salut, la volonté de retrouver l'unité de l'être pour surmonter l'angoisse destructrice. Elle sera attentive aussi aux limites qu'impose à l'homme sa finitude, aux signes d'aliénation qui enferment l'homme dans son "ipséité ». Elle s'interrogera enfin sur les signes de salut, sur les possibilités de retrouver l'unité de l'être de l'homme, l'être Nouveau, que laisse entrevoir le texte. En d'autres termes, elle cherchera à déceler les signes d'espérance qui traversent le texte. L'approche théologique n'est pas une norme, mais un choix possible parmi d'autres, elle ne saurait par conséquent prétendre épuiser le sens $d u$ texte, elle veut simplement apporter une contribution à la construction du sens inépuisable que recèle ce texte.

\section{Le désir de salut}

7 Noah est un homme riche, il a des serviteurs, un cheptel important (SÜ 327) ${ }^{14}$. Il a su s'affirmer dans la vie par la création de richesses qu'il considère néanmoins comme un don de Dieu («Gott hat sie mir gegeben » SÜ 328). Répondant à l'injonction divine de dominer la terre et les bêtes $(\mathrm{Gn} 1,28)$, il participe en quelque sorte par ses richesses à la création elle-même. Lors de sa première apparition sur scène, il exprime son plein accord avec la création, l'aube elle-même lui apparaît comme un hymne de gaieté et de reconnaissance adressé à Dieu (SÜ 325), il insiste sur l'accord intime qu'il ressent entre lui et la nature (SÜ 325). Ce sentiment de communion profonde avec la création, de participation à la création elle-même ${ }^{15}$ le conduit naturellement à exprimer sa reconnaissance envers le créateur, c'est pourquoi, contrairement au texte biblique, Noah s'apprête à offrir un sacrifice d'action de grâce avant même d'être entré dans l'arche (SÜ 325). Noah est un homme dont la réussite matérielle n'a pas altéré la profonde piété. Il s'en remet totalement au créateur («Gott behüte uns» SÜ 326) acceptant pleinement sa condition de créature. Ce qui le remplit de joie et de reconnaissance (SÜ 332), lui assure la paix intérieure et le rend enthousiaste, c'est la conscience d'être relié au fondement de l'être, d'accéder à l'unité de l'être. La participation à l'être même ouvre une dynamique qui surmonte le non-être. L'attitude de Noah au début de la pièce manifeste ce que Paul Tillich nomme le " courage d'être " qui est l'affirmation de soi «en dépit» du non-être qui le menace ${ }^{16}$. Il prend sur lui l'angoisse destructrice et l'intègre à son affirmation de soi.

8 L'expérience de l'unité intérieure est transformante, elle a une répercussion sur la relation avec les autres êtres. Noah accueille les trois voisins avec la même proverbiale hospitalité qui caractérisait Abraham aux chênes de Mambré (Gn 18, 1-15). Il accueille pareillement le mendiant, dans lequel il reconnaît son père, fait tuer pour lui l'agneau et demande qu'on lui prépare le meilleur morceau de viande (SÜ 348). Il est significatif que Noah soit le seul personnage de la pièce à manifester, au moins à certains moments, un tel sens de l'accueil de l'autre. La relation harmonieuse que Noah est capable d'établir avec les autres traduit symboliquement que le courage d'être en participant ouvre de nouvelles possibilités de vivre ensemble en société et est un 
facteur d'humanisation des relations sociales. Nous verrons plus loin aussi les limites de cette attitude.

9 La confiance fondamentale que Noah met dans son créateur, et qui est la conséquence d'une rencontre personnelle avec Dieu, lui permet de recevoir comme un don les événements positifs comme les événements négatifs de la vie. En présence des trois voisins, il reconnaît que ses richesses lui viennent de Dieu (SÜ 328), mais quand il apprend le malheur qui vient de s'abattre sur lui, il ne renonce pas pour autant à louer Dieu («Gott soll dennoch gelobt sein» SÜ 328), à le servir («ich diene ihm auch bei der Dürre» SÜ 342) et à lui offrir le sacrifice (SÜ 329). Le malheur vécu par Noah n'entame pas sa fidélité à Dieu. Le soi reste enraciné dans le fondement de l'être, cette unité maintenue donne à Noah la force d'affronter le malheur, d'intégrer les négativités de l'existence. Il y a de la noblesse dans sa manière d'accueillir le malheur (SÜ 330). Nous verrons plus loin que sa piété filiale comporte aussi un revers.

10 La scène de la tentation de Noah par Calan, qui renvoie à la tentation de Jésus au désert, met en évidence chez Noah la fermeté de l'enracinement du soi ${ }^{17}$ en Dieu, en la puissance de l'être lui-même, dans laquelle il voit la source de son être («Ihm verdanke ich, daß ich bin» SÜ 332). C'est parce que Noah perçoit Dieu comme le seul maitre de la vie et de la mort (sÜ 341), qu'il refuse le sacrifice humain (sÜ 341) que lui propose Calan pour faire venir la pluie. Jusqu'au bout Noah observe ce qu'Albert Schweitzer nommait le principe du « respect de la vie ». C'est pourquoi il refuse aussi de tuer Calan (Dein Leben ist in Gottes Hand SÜ 378) au moment où celui-ci le lui demande.

11 Enfin le courage d'être qui assume l'angoisse du non-être se manifeste dans la décision de Noah de renoncer à tous ses biens, de donner son troupeau à Calan (SÜ 351) et de partir dans la montagne (SÜ 351) pour construire l'arche comme le lui a demandé le mendiant (SÜ 350). Cette prise de décision, difficile pour Noah, montre qu'il a trouvé la force de renoncer à chercher un appui en lui-même, en ses biens, et de s'appuyer uniquement sur le Dieu. Significativement, la décision de Noah se traduit par un changement de lieu, Noah abandonne l'horizontalité pour la verticalité, ce mouvement ascendant peut être compris comme une expression symbolique du dynamisme qui surmonte le non-être. La sérénité («Noah heiter» SÜ 251) qui entoure la prise de décision est par ailleurs le signe que l'unité intérieure est préservée, qu'il y a un accord profond entre le soi et le fondement de l'être. Cependant, pour positive qu'elle apparaisse, la décision de Noah n'est pas sans ambiguïté. Le courage d'être qu'il manifeste aboutit en effet à la perte du "soi ", à l'oblitération du pôle subjectif au profit de l'Autre. C'est précisément ce qu'exprime Noah lui-même en ces termes: « Dieu est Tout, le monde est plus minuscule que le néant... » (SÜ 351 ; 358).

Calan, lui aussi, manifeste une forme de courage, le courage d'être soi-même, compris comme « l'affirmation du soi en tant que tel, autrement dit d'un soi distinct, centré-ensoi, individualisé, incomparable, libre et se déterminant $\aleph^{18}$. Si Calan apparaît tout au long de la pièce comme le blasphémateur, le tentateur de Noah et l'adversaire irréductible du Dieu justicier de l'Ancien Testament, il ne cesse pourtant de chercher un Dieu. Il exprime, lui aussi, le désir de retrouver l'unité de son être. Il revendique, lui aussi, une filiation divine ${ }^{19}$, certes différente de celle de Noah. Calan ne veut pas d'un Dieu qui impose à l'homme sa loi, exige la soumission et suscite en lui la crainte. L'affirmation de soi semble chez lui assez forte pour intégrer l'angoisse et surmonter le désespoir dont l'expression est le symbole de la colère de Dieu ${ }^{20}$, qu'il rejette avec véhémence. Calan ose affirmer avec force sa liberté, son autonomie, sa souveraineté. Il 
revendique sa réussite matérielle comme le résultat de son labeur. En ce sens, il est un enfant de 1'Aufklärung. Sa revendication d'être libre et Seigneur («frei wie er - Herr wie er» SÜ 324), cette sorte de volonté de puissance qu'il ne cesse de manifester, a par ailleurs aussi des accents nietzschéens. Nous verrons plus loin les limites de cette affirmation de soi, car elle conduira à l'hubris.

Face à la perspective de son anéantissement et en proie à la souffrance, dans laquelle il voit un châtiment mérité (SÜ 381), expression de son sentiment de culpabilité, Calan continue à rechercher l'union avec la puissance de l'être lui-même, il veut pénétrer le fondement de l'être, accéder à une autre vision de Dieu. Il s'engage sur la voie mystique. L'expérience mystique suppose un renoncement au soi, au pôle de l'individualisation au profit du pôle de la participation. Elle lui donne la force de traverser le leurre des apparences pour affirmer la supériorité de son propre être essentiel sur les éléments de non-être. Ainsi il surmonte l'angoisse du destin et de la mort et accepte sa propre fin sans révolte. Dans l'expérience mystique le soi renonce au monde des apparences pour s'unir à l'être. Ce renoncement trouve une traduction symbolique dans la situation nouvelle de Calan : rongés par les rats, il est complètement défiguré, il a perdu sa forme visible, son identité ancienne significativement Noah ne le reconnait pas -, frappé de cécité, il a perdu de vue son monde, mais cette cécité ouvre un autre monde ${ }^{21}$, il est saisi par une illumination intérieure. Calan voit un autre Dieu qu'il décrit en ces termes: "Mais moi, je vois l'autre Dieu, celui dont on dira: le monde est grand et Dieu est plus minuscule que le Néant - un petit point, une faible lueur, et Tout commence en lui, et Tout finit en lui. Il est sans forme ni voix » (SÜ 383). Cette description est diamétralement opposée à celle de Noah dont le Dieu est grand et le monde minuscule. Jusqu'au bout Calan reste un adversaire irréductible du Dieu de Noah, le Dieu qu'il découvre dans l'expérience mystique est un Dieu autre que le Dieu personnel de Noah : « En moi aussi Dieu grandit, et avec moi il continue de se transformer en quelque chose de nouveau - comme c'est beau, Noah, que moi non plus je ne sois plus une forme, mais seulement flamme et abîme en Dieu - déjà je sombre vers lui - Il est devenu moi et je suis devenu Lui - Lui avec ma bassesse, moi avec sa grandeur - une seule entité " (SÜ 383). L'expérience mystique de Calan a des accents panthéistes indéniables ${ }^{22}$. L'identification du soi à Dieu conduit à surmonter le dualisme et à retrouver l'accord profond avec soi-même et avec le fondement de l'être. Dans la fusion avec Dieu le soi s'éprouve comme divin. On ne peut comprendre cette position que sur l'arrière-fond du panthéisme qui identifie Dieu et la Nature : Calan peut affirmer la divinité de l'homme, la coïncidence de l'homme et de Dieu, en raison de ce principe panthéiste, défendu notamment par Giordano Bruno ${ }^{23}$, qui veut qu'en chacune des parties de l'univers il y ait présence universelle totale de l'être unique qui est Dieu. On comprend dès lors l'irréductibilité de l'opposition entre Calan et Noah: Calan renverse la transcendance de Dieu et le dualisme de l'être. Le monisme panthéiste permet certes de surmonter les difficultés platoniciennes d'un dualisme qui a du mal à rendre compte de la relation entre le monde concret et le monde de « l'au-delà ». Mais on voit bien la limite de ce courage d'être en participant: l'expérience mystique aboutit à la perte de soi, à l'oblitération du pôle subjectif, à la dissolution du soi dans un principe supérieur identifié à Dieu. On notera toutefois que l'expérience mystique permet à Calan de surmonter momentanément l'angoisse liée à la finitude. Contrairement à Chus, il n'exprime aucune épouvante devant l'approche du déluge, mais semble éprouver une sorte de joie sereine à l'idée qu'il rejoindra bientôt le feu de Dieu « sans forme et sans voix » (SÜ 383). 
14 Noah comme Calan expriment à des degrés divers et chacun à sa manière les deux formes de courage d'être que distingue Paul Tillich : le courage d'être en tant que soi et le courage d'être en participant ${ }^{24}$. Ce « courage d'être s'enracine nécessairement dans une puissance d'être qui est plus grande que la puissance du soi propre et que celle de son monde $»^{25}$. La préoccupation ultime que manifestent Noah et Calan atteste ainsi que le soi reste en relation avec la réalité ultime qui est le fondement de l'être. Mais le fait que ni l'un ni l'autre ne parvienne à réunir en même temps les deux formes de courage d'être, les pôles de l'individualisation et de la participation, montre que dans les conditions de finitude et d'aliénation où se trouve l'homme, ce qui est essentiellement un se trouve existentiellement désuni. La séparation des deux formes de courage fait que l'angoisse existentielle ne peut être véritablement intégrée et surmontée et qu'elle reste destructive pour l'homme. Noah et Calan oscillent en réalité entre la perte de soi et la perte du monde. Le désir de salut est marqué par l'ambiguïté. Dans les conditions de l'existence, la face lumineuse est inséparable de la part d'ombre qui peut éventuellement obscurcir complètement l'horizon, comme cela semble être le cas dans Die Sündflut, et plonger l'homme dans le désespoir. Sous l'effet de l'angoisse, le désir de salut se pervertit et se retourne contre Dieu.

\section{Les signes de l'aliénation}

15 «Faire l'expérience de l'existence comme existence signifie être dans l'angoisse ${ }^{26}$. Dans l'expérience de l'angoisse, Dieu n'est plus la source de vie, mais devient la source d'une peur mortelle. Son image complètement déformée devient insupportable à l'homme qui le perçoit désormais comme le concurrent et l'ennemi ${ }^{27}$. La destruction de l'harmonie entre Dieu et l'homme entraîne la destruction de l'harmonie entre les hommes. Les personnages de Die Sündflut illustrent cet état d'aliénation de l'homme séparé de son fondement.

16 Le titre comme l'apparition du premier personnage sur la scène révèlent d'emblée ce qui caractérise l'expérience existentielle : la prise de conscience de la finitude, de la culpabilité, de l'aliénation, de l'absurde. Pour le titre, Barlach n'utilise pas le terme «Sintflut», qui est celui de la Bible, mais le terme «Sündflut» qui renvoie à une étymologie populaire soulignant que la punition divine est la conséquence directe $\mathrm{du}$ péché. Le choix de ce titre place d'emblée le récit sous les signes symboliques du péché et de la punition ou en termes non religieux de l'aliénation et de la culpabilité. Le premier personnage qui apparaît sur scène est un lépreux bossu, symbole de l'humanité défigurée, infirme, malade, s'acheminant vers la mort, expression de la finitude de l'homme. Il est sans cesse présent dans la pièce comme une basse continue et finira par être enchaîné à Calan, l'adversaire de Dieu, comme pour lui signifier l'inéluctabilité de la finitude. Le monologue du lépreux révèle une parodie de la trinité («Ich - lieber Buckel - lieber Aussatz» SÜ 321). Cette manière ironique de considérer sa situation constitue d'emblée une critique du Dieu trinitaire de la Bible, présenté comme celui qui fait don à l'homme de sa maladie (SÜ 321) et qu'il faudrait pour cela honorer en signe de reconnaissance (SÜ 321). Le fait de poser dès le départ la question du créateur à travers sa créature ratée souligne l'absurdité d'un tel Dieu. Significativement, le lépreux bossu ne reconnaît pas Dieu qui lui apparaît sous la forme d'un voyageur distingué accompagné de deux anges («Da kommt jemand, angezogen wie ein Nichtstuer» SÜ 321), rappelant l'apparition de Mambré (Gn 18,1-15). Cela 
traduit symboliquement la rupture de l'unité entre le soi et le fondement de l'être d'où surgit le « désir illimité de tirer à soi la totalité de la réalité »"

Ce désir illimité, que la théologie nomme "concupiscence", se manifeste chez plusieurs personnages. Il concerne aussi bien «la faim physique que la satisfaction sexuelle, aussi bien la connaissance que la puissance, aussi bien la richesse matérielle que les valeurs spirituelles $»^{29}$. Avec «ses désirs débridés » (SÜ 357), Zebid incarne la tentation de la luxure, à laquelle succombent Ham et Japhet. Mais le pieux Noah n'échappe pas non plus à la tentation de la concupiscence comme en témoigne la discussion avec son épouse Ahire au sujet d'Awah, cette jeune et belle femme, qu'il a reçue en cadeau de la part de Calan. Il est si subjugué par son charme, qu'il est tenté de la garder pour lui. Ce n'est que devant l'opposition résolue d'Ahire qu'il accepte à contre cœur de la lui céder. Mais même après l'avoir cédée, il reste obsédé nuit et jour par la jeune femme (SÜ 337). Le désir de possession se manifeste également au plan de sa relation à Dieu. Le désespoir et la colère qui s'emparent de lui lorsqu'il constate que les deux anges lui ont échappé et que ce n'est pas lui mais Awah qui a été choisie pour recevoir le message de Dieu (SÜ 340) s'expliquent par ce besoin de possession. La piété de Noah se révèle prétentieuse.

La recherche d'une abondance sans limite apparaît par ailleurs comme la motivation essentielle des trois voisins qui s'opposent à la famille de Noah. Considérant Noah comme un concurrent insupportable dans l'espace limité des pâturages, ils viennent lui annoncer qu'ils ont tué ses serviteurs (SÜ 328-329) et se sont emparés de ses troupeaux (sÜ 328). Contrairement à Noah, ils ne manifestent aucun forme de piété, ils restent centrés sur eux-mêmes. Ainsi se dessine une image des relations sociales marquées par la concurrence impitoyable, la violence, l'exclusion. Les agissements des trois voisins montrent que le manque fondamental de l'existence conduit l'homme, séparé du fondement de l'être, à chercher un appui en lui-même et à justifier son existence par la négation de l'autre. En d'autres termes, ils confirment que sans la certitude d'un fondement absolu de l'existence humaine, les relations entre les hommes dégénèrent en luttes fratricides ${ }^{30}$.

Calan manifeste l'hubris dans toute sa dimension tragique. Il s'oppose avec véhémence à Dieu et aux hommes, ses relations avec les autres sont toujours des relations d'intérêt, de concurrence et de domination. Calan ne veut être que sa propre mesure. Il est totalement un homme de la terre, centré sur lui-même et son monde (SÜ 323). C'est pourquoi il ne voit pas, sinon de manière ironique (SÜ 323), l'intérêt de remercier un Dieu prétendument faible, inefficace et jaloux, de le prier et de l'aimer (SÜ 323). Contrairement à Noah, Calan ne considère pas sa richesse comme un don de Dieu. Cynique, il reconnaît avoir volé les chameaux à un homme lui-même voleur de chameaux! (SÜ 323). Son mode d'être, c'est la violence (SÜ 323). Sa richesse n'est qu'usurpation. Sans toutefois remettre en question l'existence d'un Dieu, Calan considère que celui-ci fait partie d'un autre univers et qu'il n'y a pas de communication possible entre Dieu et l'homme (SÜ 323). Cela ne l'empêche cependant pas de proposer un sacrifice humain à Noah pour faire venir la pluie (SÜ 341) et de reconnaître cyniquement que la prière est une bonne chose s'il peut en tirer un avantage (« wenn es hilft, ist Beten eine gute Sache SÜ 341). Calan ne peut imaginer un autre Dieu qu'un Dieu utile. La motivation de son comportement est exclusivement son intérêt personnel. Il est par ailleurs significatif que Calan, contrairement à Noah, ne perçoive pas le malheur qui s'abat sur son troupeau (SÜ 324) comme le signe de la colère de Dieu, 
alors même que la forme de ce malheur renvoie très clairement aux plaies d'Egypte (Ex 8). Dans son malheur, il ne fait aucune référence à Dieu. Face à Noah, Calan revendique la filiation d'un Dieu autre, de celui qui « lui a donné la force de n'être pas un serviteur " (SÜ 346). Il refuse, en somme, la condition de créature dépendante et soumise. Tout au long de la pièce, les agissements de Calan montrent clairement que, contrairement à Noah, il se détourne du centre divin auquel il appartient pour se fixer sur son propre soi. Même sa quête d'un autre Dieu se révèle n'être en définitive qu'une justification désespérée de soi-même. Il reste enfermé dans le cercle de «l'ipséité ».

Après que Noah a perdu ses serviteurs et ses biens, Calan se présente en sauveur, il lui restitue son troupeau et lui propose même d'évincer les trois voisins (SÜ 332), alimentant ainsi le cercle infernal de la violence sociale, mais en contrepartie il demande à occuper la place de Dieu auprès de Noah ${ }^{31}$. Cette scène, qui n'est pas sans rappeler la tentation de Jésus au désert (Mt 4, 8-10), indique clairement que Calan est une figure démoniaque, sorte d'ange déchu qui n'aura de cesse de s'ériger en dieu ${ }^{32}$.

A Noah qui refuse le sacrifice humain et affirme que seul Dieu est maitre de la vie et de la mort, Calan rétorque que c'est lui le maître tout puissant ${ }^{33}$. Défiant le Dieu de Noah, il moque sa faiblesse et son inefficacité (SÜ 331) et prétend démontrer la supériorité de son propre pouvoir en faisant couper les mains du jeune berger ${ }^{34}$. Après quoi, Calan se livre, dans un acte blasphématoire, à une parodie de la crucifixion en faisant clouer les mains coupées sur un poteau («nagle sie hier an den Posten» SÜ 343). Se laissant aller à une sorte de provocation jubilatoire, il retourne l'impuissance de Dieu contre Dieu luimême (SÜ 344). Aux yeux du blasphémateur et de l'usurpateur, la grandeur de Dieu est inversement proportionnelle à l'idée que lui, Calan, se fait de l'homme. C'est pourquoi dans un geste symbolique, il fait enfermer dans un sac un lutin qu'il est seul à voir et qu'il identifie au Dieu de Noah (SÜ 345). Jusqu'au bout Calan reste persuadé qu'il est plus fort que le Dieu de Noah ${ }^{35}$. Sa décision de faire brûler l'arche, pour satisfaire son besoin infini de démontrer sa supérioritée ${ }^{36}$, montre à quel point l'hubris est une structure démoniaque aux conséquences tragiquement autodestructrices. Calan reste de bout en bout un adversaire irréductible de Dieu et des hommes, dominateur et destructeur. Les autres personnages ne comptent à ses yeux que pour autant qu'ils servent ses intérêts. Les autres ne sont pour lui que des moyens. Le berger est utile pour le sacrifice (SÜ 341), Chus, son serviteur, est utile pour exécuter les basses œuvres (SÜ 343), Zebid est utile pour l'aider à enterrer Chus (SÜ 372). Noah lui sera utile pour l'aider à échapper au déluge. Sans aucun scrupule, il sollicitera une place dans l'arche (SÜ 373).

Calan n'abandonne pas pour autant l'idée de triompher de Noah et de l'asservir ${ }^{37}$. Même sauvé du déluge, il continuerait à lutter contre ce Dieu et contre Noah. Jusqu'au bout, il refuse de se laisser désarmer (SÜ 377), manifestant ainsi qu'il n'a en rien abandonné sa prétention à la domination. En parodiant cyniquement l'attitude de Noah (sÜ 373), Calan utilise toujours le même argument, faisant du triomphe de sa force et de sa volonté humaines les signes de la volonté de Dieu (SÜ 377). Il dénonce ainsi par l'absurde l'inanité du Dieu de Noah. L'irrépressible besoin de domination fait qu'à peine entré dans l'arche Calan exerce son ascendant sur toute la famille de Noah (SÜ 374) au point que les fils projettent de le tuer (SÜ 374) et que Noah l'exclut finalement (SÜ 373). Mais même cette exclusion ne mettra pas définitivement fin à l'action destructrice de Calan, car il révèle qu'Awah est enceinte de lui (SÜ 341), elle emporte donc dans l'arche son esprit de négation. 
Quand Calan comprend enfin qu'il ne pourra échapper au déluge, aux forces de destruction, il demande à Noah de le tuer (SÜ 376). Au nom de son autonomie et de sa liberté, il revendique le libre choix de sa mort, voulant ainsi rester maître de son destin et s'adonnant à l'illusion de garder le pouvoir sur Dieu et sur lui-même ${ }^{38}$. Devant l'inéluctabilité du destin, la vie elle-même perd toute sa valeur, Calan préfère l'autodestruction. En cet instant, il semble avoir perdu la dimension de l'ultime, l'insécurité, devenue absolue, l'amène à désespérer purement et simplement de la possibilité d'être. L'attitude de Calan montre finalement que sa stratégie de défense pour surmonter l'angoisse et le désespoir est auto-destructrice. Le conflit inéluctable entre ce qu'il est de manière potentielle (son essence) et ce qu'il est effectivement (son existence) dans l'entrelacement de la liberté et de la destinée le conduit à éprouver le sentiment d'une situation sans issue. La mort apparaît alors comme le seul moyen d'échapper à son soi. Le désir de mourir révèle que le courage d'être est finalement vaincu par le non-être, et donc que sa stratégie d'affirmation de soi, en se confondant avec la présomption destructrice, aboutit à l'échec.

Si c'est bien Calan qui manifeste le plus explicitement l'hubris avec toutes ses conséquences destructrices, Noah, le pieux, n'est pas épargné par cette forme de présomption qu'est l'hubris spirituelle. Face aux provocations de Calan, la confiance béate en Dieu et l'insistante soumission à sa volonté (SÜ 344) apparaissent comme parfaitement inhumaines. Par son refus d'empêcher l'atroce mutilation, Noah devient complice du méfait. Aveuglé par sa bonne conscience et sa certitude d'être un élu de Dieu $^{39}$, convaincu que les événements lui donneront raison (SÜ 366), Noah estime inutile de se justifier auprès de Calan. L'imperturbable détenteur de la vérité, qui refoule toute espèce de doute, voit dans l'approche du déluge le nécessaire accomplissement de $\mathrm{l}^{\prime}$ œuvre de Dieu ${ }^{40}$. Il ne cesse d'inviter à la confiance en son Dieu tout-puissant et juste ${ }^{41}$. Noah fait du respect de la loi de Dieu un absolu, il succombe à l'irrésistible tentation du légalisme. Il prétend en fait surmonter l'état d'aliénation par le sérieux de son obéissance à la loi qui commande.

26 Après la mutilation du berger, ce n'est pas la souffrance du malheureux qui lui importe mais la sienne provoquée par les cris de ce dernier, c'est pourquoi il prie Calan de tuer le berger pour n'avoir plus à entendre les hurlements de douleur (SÜ 343). Noah reste centré sur lui-même. En un geste qui rappelle celui de Ponce Pilate (SÜ 346), il signifie clairement son refus de toute responsabilité dans ce qui s'est passé sous ses yeux. Sa certitude de détenir la vérité le rend aveugle aux atrocités humaines. A cet instant, son attitude religieuse apparaît comme pure idéologie, comme un système d'autojustification et de sécurisation. Plus tard en présence du berger, Noah continue de rejeter avec insistance toute responsabilité et met Calan en accusation (SÜ 355-356). Sans apparemment avoir conscience du caractère inhumain et révoltant de son attitude, il insiste sur le fait que la mutilation est la volonté de Dieu ${ }^{42}$. Pour sa défense, Noah, inébranlable, se contente de rappeler la nécessité de se soumettre à Dieu, de le servir, de le craindre et de l'aimer ${ }^{43}$. La confrontation avec le berger mutilé jette une lumière très négative sur l'ostentation hypocrite de vertu et de piété de Noah. Sa piété est si aveugle qu'elle l'empêche de reconnaître dans les conseils du voyageur-Dieu (SÜ 360) la sagesse divine et qu'il en vient à considérer le mal comme la volonté de Dieu, rejoignant ainsi la position de son adversaire Calan $!^{44}$ Ici apparaissent les conséquences perverses d'une interprétation littérale de la toute-puissance de Dieu. En réalité, Noah a érigé la construction de l'arche, qui sauvera le juste, en un absolu auquel il est prêt à 
tout sacrifier. C'est pourquoi, passant outre aux conseils du voyageur-Dieu, il sollicite l'aide de Calan pour faire entrer Zebid, la païenne, dans l'arche et s'assurer ainsi la coopération de Japhet qui menaçait de mettre en péril toute l'entreprise (SÜ 357). Le voyageur-Dieu impuissant et désabusé (SÜ 361) ne peut que constater l'échec de son œuvre (SÜ 361).

La certitude de Noah de détenir la vérité durcit son cœur et le rend insensible à la souffrance humaine. Il expulse sans ménagement hors de son espace le lépreux et le berger mutilé (SÜ 355). Toute la nuit, il entend les gémissements de Calan et du lépreux (SÜ 382) sans leur venir en aide. Devant l'horrible spectacle des deux hommes rongés par les rats et défigurés (SÜ 382), Noah continue imperturbablement de considérer comme juste l'œuvre de Dieu ${ }^{45}$. Sa certitude est telle que même l'horreur la plus abjecte ne parvient pas à éveiller chez lui, comme chez Job par exemple, l'ombre d'un doute sur la justice de son Dieu. Sa piété aveugle justifie les pires horreurs. Noah ne trouve pas de mots de compassion pour ses semblables, mais, à la manière du psalmiste (Ps 4, 7, 11, $18,25)$, il se réfugie dans la certitude que Dieu pourvoira à son bien ${ }^{46}$. Jusqu'au bout et en dépit de tout ce qui arrive, Noah affirme son attachement indéfectible à un Dieu immuable, finalement monstrueux («Gott, der unwandelbare von Ewigkeit zu Ewigkeit» SÜ 383). L'hubris spirituelle de Noah consiste à ériger sa vertu limitée en vertu absolue. Cet orgueil abominable aux conséquences tragiques montre à quel point Noah, lui aussi, dans sa piété même, reste enfermé dans le cercle de «l'ipséité » et perd de vue son monde $^{47}$.

Les paroles et les actes de Calan et Noah, dans leur ambiguité, montrent que l'aliénation existentielle qui est angoisse pousse l'homme soit à se fixer désespérément sur le fini et à rejeter l'infini, soit à se cramponner à l'infini et à dévaloriser le fini. Cela aboutit dans l'un et l'autre cas, tour à tour, à la perte de soi ou à la perte du monde. Calan et Noah démontrent que toutes les tentatives pour surmonter l'aliénation à partir du pouvoir de sa propre existence aliénée conduisent l'homme à un pénible labeur et à un échec tragique. L'homme est en somme incapable d'échapper par lui-même à l'aliénation existentielle. Les trajectoires suivies par Calan et Noah laissent entières les questions fondamentales posées par la pièce : qu'est-ce qui permet de triompher de l'angoisse du doute et de l'absurde ? Sur quoi peut s'appuyer le courage de la confiance qui affirme le soi individuel sans risquer la perte de soi ni la perte de son monde? Comment sortir de l'aliénation existentielle? Comment trouver l'image juste de Dieu et la relation juste à Dieu?

\section{La manifestation de l'Être Nouveau}

Il y a dans la pièce un personnage qui, de notre point de vue, pourrait indiquer une possible voie de salut et constituer un signe d'espérance face à l'échec auquel conduisent les tentatives de salut par soi-même de Calan et de Noah. C'est le berger que Calan a capturé (SÜ 341) et auquel il fait couper les mains. La présence de ce jeune berger retient notre attention en raison de la connotation symbolique particulière que cette figure revêt dans la Bible. L'Ancien Testament identifie Yahvé au Berger : il est le pasteur de Jacob (Gn 48, 15) et d'Israël (Gn 49, 24; Ps 23 ; Ps 80), dont le peuple est le troupeau (Ps 74 ; Ps 77; Ps 79 ; Ps 95 ; Ps 100). La figure du Dieu-Berger traverse tout l'Ancien Testament. La bonté de Dieu pour son peuple est souvent comparée aux soins attentifs dont un bon berger entoure ses brebis. Dans le Nouveau Testament, Jésus 
porte l'image à son plein épanouissement quand il déclare être lui-même le bon pasteur, promis par Dieu, qui donne sa vie pour ses brebis (Jn 10) : «Je suis la porte des brebis [...] si quelqu'un entre par moi, il sera sauvé, il ira et viendra et trouvera de quoi se nourrir. (Jn 10, 7- 9). Dans l'Evangile selon Saint Jean, Jésus est le lieu de la découverte et de l'accueil des dons divins, il sauve l'homme de l'aliénation et de la mort et lui apporte la liberté et la sécurité existentielle. Même si, dans la pièce, le jeune berger ne revendique pas explicitement une mission christique, on peut néanmoins, compte tenu de la charge symbolique particulière que revêt cette figure dans la Bible et de l'attitude paradoxale que manifeste le berger dans la pièce, se demander s'il n'est pas au fond une figure christique implicite manifestant l'Être Nouveau qui surmonte l'aliénation existentielle et s'il n'apporte pas ainsi la vraie réponse aux questions posées par Calan et Noah.

Par la parodie de crucifixion (SÜ 343) à laquelle se livre Calan, le berger est de fait assimilé au Crucifié. Par ailleurs son attitude devant le "sacrifice» rappelle étrangement celle du Christ devant ses bourreaux. Significativement aucune indication scénique ni aucune parole ne révèle chez le berger des signes de protestation ou de résistance face au bourreau. Il reste silencieux. Cette attitude de docilité rappelle celle du serviteur souffrant et de l'Agneau pascal, la victime sacrificielle par excellence, dont, en référence à Isaïe 53,7, il est dit dans les Actes des apôtres: "Comme une brebis, il (Jésus) a été conduit à la boucherie ; comme un agneau muet devant celui qui le tond, ainsi il n'ouvre pas la bouche » (Ac 8,32). La docilité du berger l'apparente au Christ que par ailleurs la Bible identifie tour à tour au berger et à l'agneau. L'intention manifeste de Calan est certes blasphématoire, mais, à la manière de Ponce Pilate présentant Jésus aux grands prêtres avec ces mots "Voici l'homme» (Jn 19, 5), il désigne involontairement, par la référence à la crucifixion, la figure de l'Être Nouveau qui révèle le vrai visage du Dieu de la Bible et surmonte dans les conditions de l'existence l'aliénation existentielle.

31 Dans sa vie personnelle, le berger subit toutes les conséquences destructrices de l'aliénation existentielle : la violence physique de Calan, le rejet et l'exclusion par Noah (SÜ 355). Il est menacé comme Noah, Calan et les autres par l'angoisse autodestructrice, que symbolise la montée des eaux diluviennes. Mais son comportement paradoxal révèle un étonnant pouvoir de résistance aux forces négatives: contrairement au lépreux (SÜ 369), il n'éprouve aucune haine contre Dieu, ni aucun désir de vengeance contre les hommes. Repoussé et violenté par les hommes, il n'en continue pas moins de les accepter. Fuyant devant les flots, il trouve la force de compatir à la souffrance du lépreux, il accueille sa révolte et veille sur lui avec sollicitude $^{48}$. Ce qui le différencie de Noah et de Calan, c'est qu'il n'y a pas en lui de dureté de cœur, il se fait proche de l'humanité souffrante et pleure avec elle. Dans la souffrance et le dénuement le plus complet, le berger ne perd pas de vue le monde. Contrairement à Calan et au lépreux, il ne se révolte pas non plus contre Dieu, il ne lui attribue pas la responsabilité du mal, il ne croit pas que Dieu soit l'auteur du mal et ait raté sa création («Ich würde über Gott weinen, der das getan » SÜ 369). Cette attitude paradoxale envers les hommes et Dieu révèle que le berger a su préserver en lui l'unité entre le soi et la puissance de l'Être en dépit de la menace du non-être et que cette unité recèle une puissance de transformation de l'homme. Si l'on admet que le berger est une figure christique, on comprendra que son attitude paradoxale renvoie au paradoxe du message chrétien, à savoir que «dans une vie personnelle l'image de 
l'humanité essentielle s'est manifestée dans les conditions de l'existence sans être vaincue par elles $»^{49}$.

Interrogé par Calan («Was siehst du?» SÜ 370), l'esprit des temps modernes qui se dresse contre Dieu, le berger se contente de cette réponse sibylline: «La malédiction provient de l'aveuglement, mais moi je vois » (SÜ 370). Il donne ainsi à entendre que son refus de la malédiction, en d'autres termes son acceptation des hommes en dépit du fait qu'ils soient inacceptables, trouve sa source dans une vision autre de Dieu, qui s'éclaire si on met son attitude en relation avec ces paroles du Crucifié: "Père, pardonne-leur car ils ne savent pas ce qu'il font. » (Le, 23, 34). L'attitude du berger révèle un Dieu radicalement différent de celui de Calan et de Noah. Ce n'est ni le Dieu jaloux et vengeur de Noah, ni le dieu "sans forme et sans voix » de Calan qu'il « voit », mais le Dieu d'amour, en qui c'est l'amour du père et non la colère qui a le dernier mot. Le berger sait que la découverte du Dieu d'amour n'est pas une conquête mais un don. Il sait que ce Dieu ne peut être possédé, mais qu'il se laisse découvrir par celui qui a appris à se taire et à écouter. Il sait que cette expérience spirituelle se situe au-delà du langage, donc au-delà de la représentation théâtrale. C'est pourquoi il reconnaît humblement : «J'ai honte de parler de Dieu et autrefois non plus je ne parlais jamais de lui. Le mot est trop grand dans ma bouche. Je comprends qu'on ne peut Le comprendre, c'est tout ce que je sais de lui »(SÜ 370).

Le berger a été saisi par la puissance de l'Etre lui-même, il ne peut en témoigner qu'en posant par son silence même un acte de «foi absolue $»^{50}$. Il sait que toute tentative d'explication, de définition risque de se dissoudre dans la contradiction, le doute et l'absence de sens comme en témoignent les discours de Calan et de Noah. Il manifeste une foi que le doute a dépouillée de tout contenu concret. Le courage d'être dans sa forme radicale, telle que l'affirme ici le berger, révèle un Dieu qui dépasse à la fois le mysticisme et la rencontre de personne à personne, c'est le « Dieu au-dessus de Dieu » ${ }^{51}$, mystère du monde, qui apparaît dans le silence "quand Dieu a disparu dans l'angoisse du doute $»^{52}$.

Ce qui permet au berger d'accepter les hommes tels qu'ils sont et de maintenir l'unité entre le soi et le fondement de l'être, c'est ce courage de la confiance qui s'appuie uniquement sur la puissance de l'Être. Contrairement à Noah, il ne possède aucune des qualités de bonté, de sagesse, de piété, mais il a eu le courage d'accepter d'être accepté en dépit de la conscience de la culpabilité. Ainsi le pardon accepté change l'image du Dieu courroucé en image définitive du Dieu d'amour. C'est l'acceptation consciente de cette puissance d'acceptation qui le libère de l'aliénation existentielle et lui permet, en dépit de la menace des forces de destruction d'affirmer ce courage d'être qui fait la synthèse entre le courage d'être en participant et le courage d'être soi-même en les dépassant tous les deux. Le berger parvient ainsi à surmonter l'aliénation existentielle que ni Noah, le pieux, ni Calan, le mystique, n'avait réussi à surmonter. De ce point de vue, il se pourrait bien que ce soit la présence du jeune berger en tant que figure christique implicite qui apporte la petite lueur d'espérance dans les ténèbres qui dominent la pièce, justifiant ainsi le clair-obscur («trübe Helle» SÜ 382) du dernier tableau. 
question centrale de Die Sündflut n'est pas le rejet de Dieu, mais la recherche de l'image juste de Dieu et de la relation juste à Dieu. Cette ouverture sur la transcendance comme horizon de compréhension du sens de l'existence humaine, cette manifestation de la préoccupation ultime, révèle que l'homme du $\mathrm{XX}^{\mathrm{e}}$ siècle ne peut se satisfaire de l'univers technique, calculable et manipulable auquel a conduit l'essor de la société industrielle. Après avoir vécu la catastrophe de la Première Guerre mondiale, il ne peut occulter la mort et la culpabilité, il ne peut ignorer les structures de destruction de la vie personnelle et collective. Il n'arrive pas à considérer sa situation actuelle comme son état essentiel, ni à croire qu'il est capable de réaliser lui-même ses propres possibilités. En ce sens, Die Sündflut atteste le rejet de l'esprit de la finitude autosuffisante, la pièce est une réaction contre tout l'héritage culturel du XIX ${ }^{\mathrm{e}}$ siècle. Elle montre que la proclamation de la «mort de Dieu » n'a pas conduit à la disparition de Dieu dans la culture du $\mathrm{XX}^{\mathrm{e}}$ siècle, mais que la question de Dieu donne toujours à penser.

Les personnages de Die Sündflut incarnent des attitudes religieuses contradictoires, révélatrices à la fois des refus et des aspirations de l'expressionnisme. Noah cristallise la critique contre toute forme de dogmatisme et de légalisme, par conséquent contre les Eglises qui produisent des systèmes théologiques fermés. On remarquera que c'est précisément une figure biblique qui sert à exprimer le rejet de la religion établie. N'estce pas le signe que des symboles religieux anciens, pervertis en quelque sorte par l'appropriation des Eglises et des théologiens, ont perdu leur force originelle, leur pouvoir de libération, et ne sont plus perçus que comme l'expression d'un pouvoir hétéronome qui imposerait Dieu au monde comme un joug étranger. Le rejet de la forme de piété et de soumission à Dieu que représente Noah montre que Die Sündflut se situe dans la continuité de 1'Aufklärung et de son idéal d'autonomie. Il est par ailleurs révélateur que Barlach introduise dans sa pièce Calan, un personnage absent du récit biblique, pour exprimer la tendance mystique qui est une caractéristique de l'expressionnisme. Cela confirme qu'il y a une rupture dans la tradition religieuse et que le $\mathrm{XX}^{\mathrm{e}}$ siècle, loin de renoncer à Dieu, cherche des voies de salut en dehors de la tradition religieuse établie. Le mysticisme, en aidant l'homme à retrouver la certitude de Dieu, apparaît comme une réponse possible à la détresse de la situation religieuse du temps : les formes conceptuelles d'expression de Dieu ayant perdu leur force originelle, le mysticisme est la tentative de devenir un avec l'abîme divin par-delà toutes les formes. Nous avons souligné quels étaient les risques et les insuffisances de la voie mystique. Face au dogmatisme et au mysticisme, le berger mutilé semble indiquer l'attitude religieuse appropriée à la situation du $\mathrm{XX}^{\mathrm{e}}$ siècle, ce n'est ni la soumission à une loi religieuse extérieure, ni le rejet de toute transcendance, ce n'est ni l'hétéronomie, ni l'autonomie radicale, mais une attitude que Paul Tillich qualifie de "théonomie " $^{53}$ : le soi du berger s'enracine dans le fondement de l'Être sans se dissoudre ni perdre de vue le monde. Participant à la puissance de l'être qui s'affirme contre le non-être, il devient capable d'affirmer son propre soi contre les forces de destructions de l'existence et de surmonter l'aliénation existentielle. Mais le fait que le berger réalise la figure de l'Être Nouveau n'est-il pas le signe qu'au fond la Bible n'a rien perdu de sa force d'interpellation dans la culture du $\mathrm{XX}^{\mathrm{e}}$ siècle? 


\section{NOTES}

1. - Tillich, Paul : Théologie de la culture, Paris, 1968, p. 17.

2. - Ibid., p. 53.

3. - Ibid., p. 16.

4. - La Genèse $(5,29)$ rattache le nom hébraïque de Noé au terme qui veut dire « réconforter, restaurer » (nhm) et suggère par là que Dieu sauvera l'humanité par Noé.

5. - Voir article « Noé » in : Encyclopaedia Universalis, Thesaurus KR, 1995, p. 2576.

6. - Voir Bocian, Martin : Lexikon der biblischen Personen, Stuttgart, 1989, p. 399.

7. - Ernst Barlach, Barlach im Gespräch, aufgezeichnet von Friedrich Schult, Leipzig, 1948, p. 17.

8. - Couffignal, Robert: De "l'arbre au serpent" au "jeune homme en blanc». Etude littéraire de récits bibliques, Toulouse, Editions Universitaires du Sud, 1993, pp. 29-44.

9. - Mazellier-Grùnbeck, Catherine : Le théâtre expressionniste et le sacré, Bern, Berlin, Frankfurt/M, New York, Paris, Wien, 1994, p. 274.

10. - Tillich, Paul : L'existence et le Christ, Lausanne, 1980, p. 61.

11. - Ibid., p. 63.

12. - Kierkegaard définit l'angoisse en ces termes : « On peut comparer l'angoisse au vertige. On a le vertige quand on plonge le regard dans l'abîme. Mais la raison du phénomène n'est pas moins l'oeil que l'abîme; car il suffit de ne pas regarder. L'angoisse est ainsi le vertige de la liberté survenant quand l'esprit veut poser la synthèse et que la liberté, scrutant les profondeurs de sa propre possibilité, saisit le fini pour s'y appuyer. La liberté succombe dans ce vertige. [...] Au même instant, tout est changé, et la liberté se relevant se voit coupable. » Le Concept d'angoisse, Cuvres complètes, tome 7, Editions de l'Orante, 1966-1984, p. 163.

13. - Dieu apparaît sur scène sous la forme d'un voyageur distingué et d'un mendiant, mais il est en même temps ce personnage invisible auquel Noah se soumet.

14. - Nous utilisons l'abréviation SÜ pour désigner Die Sündflut in: Ernst Barlach: Das dichterische Werk. 1. Bd. Die Dramen. In Gemeinschaft mit Friedrich Droß herausgegeben von Klaus Lazarowicz. München : R. Piper \& Co Verlag, 1956, pp. 321-383.

15. - Ernst Barlach: Die Sündflut, op. cit., p. 325: «wirklich ich fühle am frischen Morgen so viel Freude, als ob die Fettigkeit des Landes von den Füßen aufwärts durch alle Glieder bis in die Brust und Kopf hinauftriebe und nun in linder leiser Lust zerflösse».

16. - Tillich, Paul: Le courage d'être, Paris, Casterman, 1967, p. 10.

17. - Paul Tillich note à ce propos dans Le courage d'être p. 92 : « Mais le soi n'est un soi que parce qu'il a un monde, un univers structuré, auquel il appartient et dont il est séparé en même temps. Le soi et le monde sont en corrélation et ainsi en est-il de l'individualisation et de la participation. » Le terme « soi » est plus englobant que le terme « ego ». On peut rapprocher cette façon de comprendre le «soi » de celle de CG. Jung qui dans L'Homme à la découverte de son âme, p. 398 écrit: «Le Soi est notre totalité psychique, faite de la conscience et de l'océan infini de l'âme sur lequel elle flotte... Ainsi le Soi est infiniment plus vaste que le Moi. S'aimer soi-même, ce devrait être aimer cette totalité, à travers laquelle on aimerait l'humanité tout entière ».

18. - Tillich, Paul : Le courage d'être, op. cit., p. 90.

19. - Ernst Barlach : Die Sündflut, op. cit., p. 346 : «ich, Calan, ein Kind Gottes, der mir die Kraft gegeben hat, kein Knecht zu sein»; «Einer war es, Noah, und ich bleibe der Sohn dieses Einen», p. 366 ; «Der Eine, Noah, dessen Sohn ich bin, der mich frei und ohne Furcht ins Leben gelassen, der hat mich vergessen.», p. 376.

20. - Tillich, Paul : L'existence et le Christ, op. cit., p. 97-98.

21. - Ernst Barlach: Die Sündflut op. cit., p. 382 : «Als die Ratten meine Augen aus den Höhlen rissen, Noah, bin ich sehend geworden. Ich ertrage den Anblick Gottes, ich sehe Gott». 
22. - Contrairement à Catherine Mazellier-Grünbeck (Le théâtre expressionniste et le sacré, p. 275), K.S. Guthke («Das Drama des Expressionismus und die Metaphysik der Enttäuschung» in: W. Paulsen (Hrg.), Aspekte des Expressionismus, p. 56) et Ch. Eykmann (Denk- und Stilformen des Expressionismus, München, 1974 p. 85) ont raison de parler du panthéisme de Barlach.

23. - Voir Seidengart, Jean : «Giordano Bruno (1548-1600)» in: Encyclopaedia Universalis. Tome 4. Paris, 1995, pp. 602-604.

24. - Voir Tillich, Paul : Le courage d'être, op. cit., pp. 91-150.

25. - Ibid., p. 151.

26. - Tillich, Paul : Aux frontières de la religion et de la science, Paris, 1970, p. 118.

27. - Voir Eugen Drewermann: Strukturen des Bösen. Teil 1. Die jahwistische Urgeschichte in exegetischer Sicht. Paderborn, München, Wien, Zürich: Ferdinand Schöningh. 1992. pp. 61-67.

28. - Tillich, Paul : L'existence et le Christ, op. cit., p. 69.

29. - Ibid., p. 69.

30. - Voir Eugen Drewermann : Strukturen des Bösen. Teil 3. Die jahwistische Urgeschichte in philosophischer Sicht. Paderborn, München, Wien, Zürich: Ferdinand Schöningh, 1992, pp. 277-278.

31. - Ibid., p. 331 : «Wie wäre es, du ließest mir einen Platz neben ihm -ließest mich walten für dich an seiner Statt?».

32. - Ibid., p. 344 : «... ich bin kein Mensch von deiner Sorte, bin das Kind eines größeren Gottes als deiner - ein Gotteskind, Noah, das abgesetzt, verloren, gestohlen, übelgehalten und verwahrlost ist - aber ein Gott!».

33. - Ibid., p. 341-342: «Er - nein sondern ich ; und ich mache dich zum Herrn. [...] soll ich nun vor dir neben Gott treten? Ich bin stark und mächtig und gnädig. Wenn er der Herr ist über Tod und Leben, so stehe ich ihm darin nicht nach. [...] Ich muß dir zeigen, daß ich gottmächtig bin.»

34. - Ibid., p. 342- 343 : «... schlage ihm beide Hände herunter. [...] Läßt er es zu, so sehe ich darin ein Zeichen, daß ihm das Opfer gefällig ist, oder daß sein Grimm gewaltlos ist gegen meine geringe Götterschaft. Dann wäre er geringer als ich. [...] Mein Wort schlägt Hände ab [...] wer, wenn nicht ich, ist der Herr?»

35. - Ibid., p. 366 : «... ich bin, der ich sagte, daß ich sein wollte: stärker als Er, und ich habe dir gezeigt, daß ich stärker bin».

36. - Ibid., p. 366 : «... hat Er Wasser in seiner Hand, so habe ich Feuer in der meinen. Dein Haus wird brennen, Noah, mein Werk wird seines überwinden.»

37. - Ibid., p. 373 : «Du wirst Herr werden über uns, Calan. Und wirst in Gottes Hause fahren und mich und meine Söhne zu Knechten machen. Du weißt es, Calan!» et p. 377 : «So fahre ich [Calan] auf der Flut und spotte deines Gottes. Ihr alle sollt meine Knechte werden.»

38. - Ibid., p. 377 : «Ich bin stärker als er, vergiß das nicht, und will sterben wie es dem Sohn ansteht, der kein Knecht seines Vaters ist.»

39. - Ibid., p. 366 : «Wie wir sind, Calan, so will uns Gott erhalten - wie Gott will, denke ich, so gut sind wir, nicht besser und nicht böser.»

40. - Ibid., p. 363 : «Die Zeit ist reif, Winde tragen die Flut heran.» et p. 365 : «Es ist nun soweit, Calan, die Zeit ist reif [...] Die Zeit ist erfüllt, [...] Gott ist am Werk.»

41. - Ibid., p. 363 : «Befehlt euch in Gottes Hut [...] vertraut auf Gott. [...] Gott hat gebaut...».

42. - Ibid., p. 355-356: «... Gott hat es zugelassen, nicht ich. [...] es sind Gottes Werke...».

43. - Ibid., p. 356 : «Betet zu Gott [...] dient ihm [...] furchtet ihn [...] liebt ihn.»

44. - Ibid., p. 361 : «Was Gott zu Wölfen werden läßt, das bellt und beißt und heult mit Recht.»

45. - Ibid., p. 382 : «Gottes Walten ist gerecht, aber seine Gewalt ist über die Kraft meiner Augen, sie ertragen nicht den Anblick seines Tuns.»

46. - Ibid., p. 383 : «Gott ist mein Hirt, mir wird nichts mangeln. Er wird mich durch die Flut führen und mich retten vom Verderben.» 
47. - Ibid., p. 356 : «Wir wollen bauen [...] wir haben keinen Platz als für uns und unsere Tiere! [...] Sie sind es nicht wert zu leben, Awah, wir allein sind es wert...»

48. - Ibid., p. 369 : «Du bist vom vielen Fluchen müde und heiser geworden [...] Alles schläft, schlafe auch, ich hüte dich. »

49. - Tillich, Paul : L'existence et le Christ, op. cit., p. 117.

50. - Tillich, Paul : Le courage d'être, op. cit., p. 169.

51. - Ibid., p. 178.

52. - Ibid., p. 181.

53. - Voir Tillich, Paul: Théologie systématique. Introduction. Raison et Révélation. Tome 1. Traduit de l'américain par Fernand Ouellet. Paris : Editions Planète, 1970. p. 170.

\section{RÉSUMÉS}

Le présent article est un essai d'approche théologique du drame Die Sündflut (1924) d'Ernst Barlach à l'aide de catégories développées par le théologien Paul Tillich : la préoccupation ultime, le courage d'être, la foi absolue, l'Etre Nouveau. L'analyse souligne que la structure antithétique de la pièce est l'expression de l'ambiguïté fondamentale de l'être de l'homme sous les conditions de l'existence, d'où résultent les visages contradictoires de Dieu et les attitudes contradictoires des hommes envers Dieu. C'est à partir de l'aliénation existentielle qui est angoisse que s'éclairent les paroles et les actes des personnages cherchant une voie de salut, soit dans l'infini, soit dans le fini. Les trajectoires des deux protagonistes, Noah et Calan, montrent cependant l'échec tragique des tentatives de salut par soi-même. Le légalisme de Noah comme l'hubris, puis le mysticisme de Calan ne conduisent qu'à la perte de soi ou à la perte du monde. Le chemin permettant de surmonter l'angoisse du doute et de l'absurde liée à la finitude est esquissé par un jeune berger, au comportement paradoxal, dont nous avons montré qu'il était une figure christique implicite réalisant l'Être Nouveau, qui surmonte l'aliénation existentielle. Die Sündflut atteste tant le rejet de l'esprit de la finitude autosuffisante hérité du XIX ${ }^{\mathrm{e}}$ siècle que la persistance de la force d'interpellation de la Bible dans la culture du XX $\mathrm{X}^{\mathrm{e}}$ siècle.

Der vorliegende Aufsatz ist ein Versuch, das Drama Die Sünflut (1924) von Ernst Barlach vom theologischen Gesichtspunkt aus zu interpretieren, und zwar mit Hilfe von Begriffen, die der Theologe Paul Tillich entwickelt hat: das unbedingte Angehen (ultimate concern), der Mut zum Sein, der absolute Glaube, das Neue Sein. In unserer Analyse unterstreichen wir, daß die antithetische Struktur des Stückes Ausdruck der grundsätzlichen Ambivalenz des Seins im Menschen unter den Bedingungen der Existenz ist, woraus sich die gegensätzlichen Gottesvorstellungen und die gegensätzlichen Stellungnahmen des Menschen zu Gott ergeben. Vor der angstbeladenen existentiellen Entfremdung erhellen sich die Worte und Taten der Gestalten, die einen Weg der Erlösung entweder im Endlichen oder im Unendlichen suchen. Die Lebenswege der beiden Protagonisten, Noah und Calan, zeigen das tragische Scheitern der aus eigener Kraft unternommenen Erlösungsversuche. Der Legalismus von Noah wie die Hybris und der Mystizismus von Calan führen entweder zum Verlust des Selbst oder zum Verlust der Welt. Der Weg zur Überwindung der mit der Endlichkeit verbundenen Angst vor Zweifel und Sinnlosigkeit wird von einem jungen, sich paradox verhaltenden Hirten angedeutet, in dem eine implizite Christusgestalt erkennbar ist, die das Neue Sein verwirklicht und die existentielle Entfremdung überwindet. In der Sündflut wird der aus dem XIX. Jahrhundert ererbte 
selbstgenügsame Geist der Endlichkeit zurückgewiesen und die ungebrochene Kraft der Bibel in der Kultur des beginnenden XX. Jahrhunderts bestätigt.

\section{AUTEUR}

\section{BERNARD BACH}

Université Charles-de-Gaulle, Lille III 